\title{
Linx
}

Revue des linguistes de l'université Paris X Nanterre

$53 \mid 2005$

Le semi-figement

\section{Léa lave son linge à la main ou comment à la main ne désigne pas la partie du corps}

\section{Belinda Lavieu}

\section{(2) OpenEdition}

Journals

Édition électronique

URL : http://journals.openedition.org/linx/279

DOI : $10.4000 / \operatorname{lin} \times .279$

ISSN : 2118-9692

Éditeur

Presses universitaires de Paris Nanterre

\section{Édition imprimée}

Date de publication : 1 décembre 2005

Pagination : 173-181

ISSN : 0246-8743

\section{Référence électronique}

Belinda Lavieu, "Léa lave son linge à la main ou comment à la main ne désigne pas la partie du corps », Linx [En ligne], 53 | 2005, mis en ligne le 15 février 2011, consulté le 30 avril 2019. URL : http:// journals.openedition.org/linx/279; DOI : 10.4000/linx.279 


\title{
Léa lave son linge à la main ou comment à la main ne désigne pas la partie du corps*
}

\author{
Belinda Lavieu \\ Université de Paris X - Nanterre et Laboratoire MoDyCo \\ (CNRS UMR 7114)
}

\section{Introduction}

On associe à la notion de figement trois contraintes d'ordre sémantique, lexical, et morpho-syntaxique. En fonction de ces critères, nous chercherons à expliquer pourquoi des énoncés comme

(1) Un brouillard à couper au couteau (" un brouillard dense et épais»)

(2) Être à ramasser à la petite cuiller («être à bout de force »)

(3) Mener quelqu'un à la baguette («commander avec autorité et rigueur»)

(4) Manger du caviar à la louche (« en grande quantité»)

sont reconnus comme figés et étiquetés par les dictionnaires - dont le TLF ${ }^{1}-$ comme "locutions », alors que des séquences telles que teindre (ses cheveux) au henné ou inciser (une plaie) au scalpel ne le sont pas. Nous essaierons alors de situer des énoncés comme

* Je remercie Danielle Leeman et Antoinette Balibar-Mrabti pour leurs remarques et suggestions.

1 Abréviation pour Trésor de la Langue Française. 
nourrir au sein/au biberon/à la cuiller ou laver à la main entre ces deux extrêmes. Ainsi, nous pouvons représenter sur un axe linéaire les différents «degré de figement» (Martin 2001) puisque c'est bien de cela dont il sera question ici.

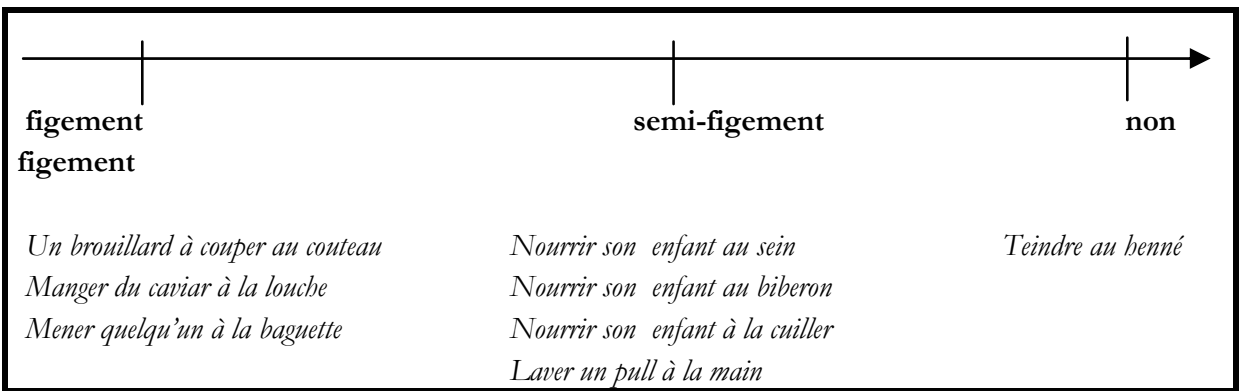

Figure 1 : Schéma des degrés de figement d'une séquence comportant un GP [moyen] introduit par la préposition à

Nous nous arrêterons en particulier sur les énoncés que nous avons provisoirement regroupés sous l'étiquette « semi-figement».

Dans la plupart des études consacrées aux expressions figées, trois propriétés sont généralement reconnues comme essentielles :

1. du point de vue sémantique : le sens de l'expression est global

2. du point de vue lexical : l'absence de variation paradigmatique (blocage lexical)

3. du point de vue morphosyntaxique: les restrictions au niveau des manipulations syntaxiques et morphologiques

mais en raison d'une terminologie diverse et abondante, il est difficile de s'y retrouver. Pour remédier à ce problème, nous présentons dans le tableau ci-après les termes employés par d'autres chercheurs et leur faisons correspondre notre propre dénomination.

\begin{tabular}{|c|c|c|}
\hline Dénomination & $\begin{array}{l}\text { Termes employés par } \\
\text { d'autres chercheurs }\end{array}$ & Nom \\
\hline \multirow[t]{3}{*}{ Non-compositionnalité } & Opacité sémantique & G. Gross (1996: 10) \\
\hline & $\begin{array}{l}\text { Restrictions sélectionnelles, } \\
\text { non-compositionnalité, valeur non } \\
\text { référentielle }\end{array}$ & R. Martin (1997 : 292-293) \\
\hline & Séquence dite « opaque» & C. Schapira (1999: 11) \\
\hline \multirow[t]{3}{*}{ Blocage lexical } & $\begin{array}{l}\text { Degré de figement, } \\
\text { Blocage des paradigmes synonymiques, } \\
\text { Défigement }\end{array}$ & G. Gross (1996 : 16-17-19) \\
\hline & Restrictions sélectionnelles & R. Martin (1997 : 292-293) \\
\hline & $\begin{array}{l}\text { L'impossibilité de remplacer l'un ou } \\
\text { l'autre des mots du groupe, } \\
\text { Eléments archä̈ques de nature } \\
\text { syntaxique (ordre des mots) }\end{array}$ & C. Schapira (1999: 9) \\
\hline \multirow[t]{2}{*}{ Restrictions morphologiques } & Non-actualisation des éléments & G. Gross (1996: 13) \\
\hline & $\begin{array}{l}\text { Eléments archä̈ques de nature } \\
\text { morphologique, }\end{array}$ & C. Schapira (1999 : 10-11) \\
\hline
\end{tabular}


'Léa lave son linge à la main' ou comment à la main' ne désigne pas la partie du corps

\begin{tabular}{|l|l|l|}
\hline Restrictions syntaxiques & $\begin{array}{l}\text { Eléments archä̈ques de nature } \\
\text { syntaxique, } \\
\text { Constructions elliptiques }\end{array}$ & \\
& $\begin{array}{l}\text { Blocage des propriétés } \\
\text { transformationnelles, non-insertion, } \\
\text { absence de libre actualisation des } \\
\text { éléments composants }\end{array}$ & G. Gross (1996:12-18-32) \\
& $\begin{array}{l}\text { Impossibilité de changer l'ordre des } \\
\text { mots dans la séquence figée, }\end{array}$ & C. Schapira (1999:9) \\
& $\begin{array}{l}\text { La suspension de la variation en } \\
\text { nombre des composantes, } \\
\text { Le segment figé n'admet pas la } \\
\text { manipulation transformationnelle, } \\
\text { Le segment figé ne permet pas } \\
\text { l'extraction d'un des composants pour } \\
\text { la relativisation, la topicalisation, la } \\
\text { voix passive ou la mise en vedette au } \\
\text { moyen de la corrélation 'c'est...que' }\end{array}$ & \\
\hline & \\
\hline
\end{tabular}

Tableau 1 : Correspondance terminologiques des critères de figement

\section{Du point de vue sémantique : le sens de l'expression est global ou non compositionnel}

L'application de ce critère dépend de la théorie du sens que l'on se donne, et donc de la définition que l'on donne aux mots. Si l'on admet que couper signifie «diviser », «séparer en deux morceaux» et que couteau désigne un instrument tranchant, on ne peut pas interpréter un brouillard à couper au couteau comme on le fait de couper le pain au couteau, car le brouillard n'est pas un solide comme l'est le pain : à strictement parler, on ne peut pas couper le brouillard au couteau pour en obtenir des tranches ou des morceaux. Cependant, si l'on cherche à définir le groupe verbal à partir d'autres emplois, comme le poisson surgelé est si dur que je dois le couper au couteau, ou comme la ficelle est trop serrée : on doit la couper au couteau, on voit que le GV entretient une relation avec la notion de « difficulté de passage ». Dans ce cas, il y a un point commun entre brouillard à conper au coutean et poisson à couper an coutean ou ficelle à couper an coutean ; de plus, la construction sert souvent à introduire le haut degré : un film à mourir de rire, être bête à manger du foin, or brouillard à conper au coutean implique aussi un haut degré (celle de l'épaisseur du brouillard). Ainsi peut-on admettre que, si couper au couteau dans un bronillard à couper au couteau n'a pas le sens de couper au couteau dans une ficelle à couper an couteau, les deux emplois ne sont toutefois pas totalement étrangers l'un à l'autre. En revanche, être à ramasser à la petite cuiller signifie que l'on est très fatigué, mais si l'on retrouve le sens de haut degré que l'on a aussi dans la construction précédente, la notion de «fatigue » ne parait aucunement présente dans ramasser le sucre en poudre à la petite cuiller par exemple. Donc être à ramasser à la petite cuiller au sens de «être à bout de forces » présente une non-compositionnalité supérieure à celle de un brouillard à couper an couteau. 
Dans ratisser ou passer la pièce au peigne fin, on peut aussi ou bien partir d'un sens étroitement dénotatif ou bien intégrer dans la définition des connotations associées à l'emploi dit «propre ». Dans le premier cas, l'expression apparaît complètement noncompositionnelle. En revanche, on peut considérer qu'il y a un lien entre le peigne fin et le souci de ne pas laisser passer le moindre détail : par exemple le peigne fin est recommandé pour repérer les poux dans une chevelure; dans ce cas, passer une pièce au peigne fin a un lien sémantique avec le sens que l'on peut considérer " premier » et par conséquent le degré de non-compositionnalité est moindre que pour je suis à ramasser à la petite cuiller, et de même, le sens est plus directement calculable que pour un brouillard à couper au couteau.

Mener quelqu'un à la baguette est lui-même plus compositionnel que passer une pièce au peigne fin, du fait que l'expression évoque assez naturellement par exemple le chef d'orchestre qui impose à ses musiciens une certaine exécution du morceau de musique ; certes une femme qui mène son mari à la baguette ne le dirige pas à l'aide d'une baguette, mais le sens du verbe d'une part, et celui de l'instrument qui n'évoque pas seulement le moyen mais aussi l'autorité, d'autre part, se retrouvent assez facilement dans l'expression dite «figurée ». Donc mener quelqu'un à la baguette est plus proche de la compositionnalité que ne l'est passer une pièce au peigne fin, lui-même plus compositionnel que je suis à ramasser à la petite cuiller.

En conclusion, on peut établir une échelle selon le degré de proximité de l'emploi dit «figé » avec les emplois dits « libres » si - comme le fait Jacqueline Picoche à propos de On n'est pas sortis de l'auberge ! par rapport à auberge - on ne borne pas le sens des mots à leur stricte désignation référentielle objective. La louche seulement définie comme une cuiller à long manche et à cuilleron semi-sphérique ne permet pas d'établir le lien avec la grande quantité dans manger du caviar à la louche; il suffit de considérer qu'une louche permet de servir des parts plus grandes qu'une cuiller pour que la relation apparaisse assez transparente. De même la pelle (dans, par exemple, ramasser des récompenses à la pelle .) est-elle définie comme ce qui permet de ramasser une grande quantité de morceaux ou de matière pulvérulentes et non pas seulement comme un certains outil formé d'une plaque et d'un manche. Du point de vue du critère de compositionnalité, donc, il y a des degrés dans le figement, c'est-à-dire dans l'intuition des rapports entre l'expression considérée et les autres emplois des mots qui la forment, mais à condition d'établir une continuité entre ces emplois et de ne pas réduire le sens de chacun à sa stricte dénotation. Ainsi, on pourrait ordonner les exemples de la manière suivante :

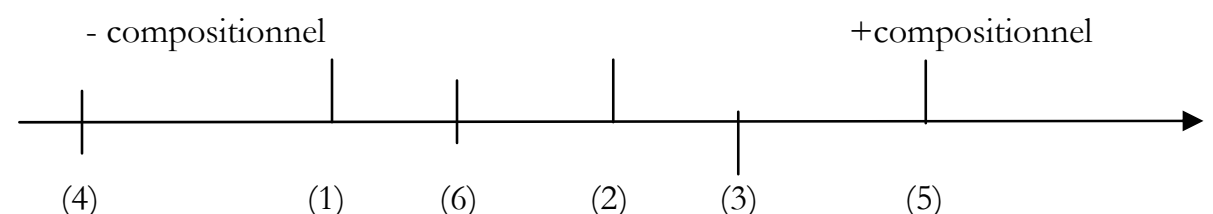

Figure 2 : Echelle de compositionnalité 
avec :

(1) Un brouillard à couper au couteau

(2) Manger du caviar à la louche

(3) Distribuer des récompenses à la pelle

(4) Etre à ramasser à la petite cuiller

(5) Mener quelqu'un à la baguette

(6) Ratisser la pièce au peigne fin

\section{Du point de vue lexical : au moins un des éléments de l'expression ne connaît pas de variation paradigmatique}

Quand il y a blocage lexical dans une expression, il est impossible de remplacer un mot lexical par un autre. Ainsi la commutation synonymique n'est pas possible, dans :

(1.a) *Une brume à couper au couteau

(1.b) *Un brouillard à (découper+tailler) au couteau

(1.c) $\quad *$ Un brouillard à couper au (canif+cutter)

ni dans :

(2.a) *Avaler du caviar à la louche

(2.b) *Manger des œufs de lompes à la louche

(2.c) *Manger du caviar à la fourchette

ni non plus dans :

(3.a) *Distribuer des récompenses à la bêche

(3.b) *Donner des récompenses à la pelle

(3.c) *Distribuer des cadeaux à la pelle

Ce critère lexical semble beaucoup moins discutable que le critère sémantique dans la mesure où les inacceptabilités sont nettes. En même temps, les résultats ne concordent pas avec le test de compositionnalité tel que nous l'avons pratiqué puisqu'en l'occurrence on ne peut plus distinguer le degré de figement.

\section{Du point de vue morphosyntaxique : les manipulations usuelles sont limitées}

On peut en particulier évoquer la restriction au niveau des déterminants, au niveau du nombre en ce qui concerne le nom (restrictions morphologiques) et des restrictions au niveau de certaines manipulations syntaxiques (passivation, pronominalisation, détachement : les restrictions syntaxiques, etc.). 


\subsection{Pour les restrictions morphologiques, on observe par exemple des contraintes}

- sur le déterminant :

(1.d) $\left(\mathrm{Un}+* \mathrm{le}+*\right.$ ce) brouillard à couper au couteau ${ }^{2}$

- sur le nombre :

(3.e) Distribuer (des+*une) récompenses à la pelle

(4.e) Mener quelqu'un (à la+*aux) baguettes

- sur la préposition :

(1.f) *Un brouillard à couper avec le couteau

(4.f) *Mener quelqu'un avec la baguette

(6.f) *Ratisser la pièce avec le peigne fin

Là encore, on est conduit à conclure que les expressions considérées sont également figées, ce qui contredit les degrés de figement au niveau compositionnel.

\subsection{Pour les restrictions syntaxiques, les manipulations concernent essentiel- lement la passivation, l'extraction, la pronominalisation}

\subsubsection{La passivation}

(1.g) *Un brouillard a été coupé au couteau

(2.g) *Du caviar a été mangé à la louche

(3.g) Des récompenses ont été distribuées à la pelle

(4.g) *Léa a été ramassée à la petite cuiller

(5.g) Il est mené à la baguette par sa femme

(6.g) La pièce a été passée au peigne fin

Les énoncés (3) et (6) se prêtent plus facilement à la passivation que les quatre autres ce qui correspond en gros au « + compositionnel » dans la figure 2.

\subsubsection{L'extraction}

La seconde manipulation syntaxique est celle de l'extraction en « c'est...que » :

(1.h) *C'est au couteau que le brouillard est à couper

(2.h) ?C'est à la louche qu'ils mangent le caviar

(3.h) C'est à la pelle qu'on distribue les récompenses

(4.h) *C'est à la petite cuiller que Marie est à ramasser

(5.h) C'est à la baguette que Marie mène ses enfants

(6.h) *C'est au peigne fin que la pièce est ratissée

Ici en revanche (1) et (4) sont impossibles et correspondent au «- compositionnel » (figure 2) et s'opposent à (2), (3), (5) et (6) qui sont eux du coté « + compositionnel ».

\footnotetext{
${ }^{2}$ Mais A. Balibar-Mrabti nous fait remarquer que serait acceptable Le bronillard était à conper an couteau.
} 
'Léa lave son linge à la main' ou comment â la main' ne désigne pas la partie du corps

\subsubsection{La pronominalisation}

Ce test ne peut concerner aucun des GP en à, mais ce serait aussi le cas dans les emplois libres. Donc le test n'est pas ici révélateur.

Nous ne procéderons pas à l'ensemble des manipulations proposées dans les différents travaux consultés, mais on peut constater que les résultats ne convergent pas : si le critère sémantique permet de classer les expressions du «-» au «+» compositionnel, le critère morphologique aboutit à une annulation du continuum ainsi établi tandis que le critère syntaxique montre bien une diversité de comportements, mais qui ne coïncide pas exactement avec l'échelle obtenue à partir du critère sémantique.

\section{Application}

Nous proposons maintenant d'analyser les exemples contenus sous (7) :

(7) a. Eve nourrit son enfant au sein

b. Marie lave son linge à la main

Contrairement aux exemples précédents, les énoncés (7) ne vérifient pas les trois critères de figement. En particulier celui d' «opacité sémantique » dans la mesure où c'est le sens de chacun des constituants qui assure le sens de l'ensemble de la séquence. Du point de vue sémantique on ne peut donc pas vraiment parler d'un bloc puisque c'est bien l'ensemble des constituants qui donne du sens à l'ensemble de l'énoncé. Dans Eve nourrit son enfant au sein/au biberon/à la cuiller, le sein, le biberon ou la cuiller sont bien des moyens explicites utilisés pour que Eve nourrisse son enfant, alors que dans les énoncés analysés plus haut, la petite cuiller, le couteau, ou la baguette ne servaient pas, n'étaient pas utilisés (au sens propre) pour, respectivement, ramasser une personne, couper le brouillard ou mener des troupes. Ce premier critère fait alors pencher l'énoncé (7.a) plutôt du côté compositionnel et donc non figé ; mais pour l'énoncé Marie lave son linge à la main, il me semble que celui-ci peut recevoir deux interprétations : la première compositionnelle - qui consiste à voir Marie les mains plongées dans une bassine d'eau savonneuse pour laver des vêtements (fragiles) et la seconde qui consiste à décrire une technique de lavage «manuelle» en opposition à un lavage en machine «automatique ». Dans ce deuxième type d'interprétation, on peut supposer que l'analyse n'est pas compositionnelle et que derrière main il y a en fait le choix d'un type de lavage en opposition à un autre : cette interprétation peut d'ailleurs être corroborée de manière empirique par le fait que certaines machines à laver prévoient dans le programme « délicat » une rubrique « lavage à la main », or c'est bien la machine qui va laver le linge en question!

Le critère de restriction lexicale semble relativement satisfait comme l'illustre (8) :

(8) a. Eve nourrit son enfant (au+à la) (sein+ biberon + cuiller ${ }^{*}{ }^{*}$ fourchette ${ }^{*}$ cuiller à soupe)

b. Marie lave son linge à la/au (main + machine $+*$ doigt $+*$ pied $)$ 
Les énoncés sous (8) ont bien un paradigme limité, dans la mesure où celui-ci se réduit à deux entrées main et machine pour (8.b) et à ce que G. Gross a appelé une "classe d'objet» en (8.a) ; la contrainte lexicale est malgré tout moins forte pour les énoncés Eve nourrit son enfant (au sein+au biberon $+a ̀$ la cuiller) ou Marie lave son linge (à la main $+a ̀$ la machine) que pour les énoncés « figés » que nous avons analysés plus haut.

Enfin, pour ce qui est de la restriction morphosyntaxique, on constate qu'au moins un des éléments des énoncés (7.a) et (7.b) ne peut subir de manipulations. Ainsi, on ne peut avoir, par exemple, pour le nombre :

(9) a. *Eve nourrit son enfant aux (deux) seins, ou Eve nourrit son enfant aux (*biberons $+*$ cuillers)

b. *Marie lave son linge aux (deux) mains/ ?à deux mains (mais dans ce cas il ne peut s'agir du lavage manuel)

ni pour l'ajout d'un modifieur :

(10) a. *Eve nourrit son enfant au sein droit

b. ??Eve nourrit son enfant au biberon de plastique

c.*Marie lave son linge à la main droite

d. Marie lave son linge à la machine électrique

Il y a donc des restrictions au niveau du nombre du $\mathrm{N}$ [moyen] et au niveau du modifieur.

Mais l'extraction serait possible, de même que le passif :
a. C'est au sein qu'elle nourrit son enfant
b. C'est à la main qu'elle lave son linge
a. Son enfant est nourri au sein
b. Son linge est lavé à la main

On se trouve bien en peine lorsqu'il faut trancher le caractère figé ou non des énoncés (7.a) et (7.b). A partir de cette analyse, on en vient à ce demander s'il n'y a pas des critères de figement qui sont plus significatifs que d'autres. Nous pouvons établir un tableau récapitulatif indiquant le caractère nécessaire ou suffisant du critère pour étiqueter la séquence de « figée ».

\begin{tabular}{|l|c|c|c|}
\hline \multicolumn{1}{|c|}{ critère } & condition nécessaire & condition suffisante & indice \\
\hline Non-compositionnalité & - & + & \\
\hline Restriction lexicale & + & + & \\
\hline Restriction morphosyntaxique & & & \\
& & - & + \\
\hline Restriction morphologique & - & - & + \\
\hline Restriction syntaxique & - & - & + \\
\hline
\end{tabular}

Tableau 2 : Résumé schématique des CNS des critères examinés 
'Léa lave son linge à la main' ou comment â la main' ne désigne pas la partie du corps

Ainsi, si je peux démontrer le caractère non-compositionnel des séquences, cela me suffit pour l'étiqueter «figée » en revanche la compositionnalité d'une séquence n'implique pas nécessairement que celle-ci ne soit pas figée, il faut dans ce cas regarder les critères d'ordre lexical et morphosyntaxique. Le premier (restriction lexicale) est à la fois une condition nécessaire et une condition suffisante pour indiquer un cas de figement : Eve nourrit l'enfant au biberon + au sein $+a ̀$ la cuiller et Marie lave son linge à la main sont donc bien figées ; quant aux critères morphosyntaxiques ils ne constituent pas un indice systématique de figement. Comme le dit A. Balibar-Mrabti (2004 : 28), "réfléchir sur le semi-figement, c'est considérer que les mécanismes syntaxiques sont à la fois ouverts et fermés ».

\section{RÉFÉRENCES}

Balibar-Mrabti, A. (2004), «Lexique-Grammaire et extensions lexicales. Notes sur le semifigement», Linguisticae Investigationes XXIV, Amsterdam/Philadelphia, John Benjamins, pp. 23-30.

BALLY, Ch. (1921), Traité de stylistique française, Paris, Klincksieck.

Danlos, L. (éd.) (1988), «Les expressions figées », Langages, 90, Paris, Larousse.

Gross, G. (1996), Les expressions figées en français. Noms composés et autres locutions, Paris/Gap, Ophrys.

Gross, G. (1997), «Du bon usage de la notion de locution », in M. Martins-Baltar (éd.). La locution entre langue et usages, Fontenay/Saint-Cloud, ENS éditions, pp. 201-225.

Gross, M. (1988), «Les limites de la phrase figée », Langages 90, Paris, Larousse, pp. 7-22.

KLEIBER, G. (1989), «Sur la définition des proverbes », in G. Greciano (éd.). Europhras 1988. Phraséologie contrastive. Strasbourg, Université des Sciences Humaines, pp. 233-253.

KLEIN, J.-R. \& Rossari, C. (2003), «Figement et variations en français de Belgique, de France, du Québec et de Suisse», Lingvisticae Investigationes XXVI : 2, Amsterdam/Philadelphia, John Benjamins, pp. 203-214.

LAmiroy, B. (2003), "Les notions linguistiques de figement et de contrainte », Linguisticae Investigationes XXVI : 1, Amsterdam/Philadelphia, John Benjamins, pp. 1-14.

MARTin, R. (1997), Sur les facteurs du figement lexical, in Martins-Baltar, M. (éd.). La locution entre langue et usages, Fontenay/St Cloud. Enc éditions.

Martin, R. (2001), Sémantique et automate, Paris, PUF.

MEL'ČUK, I. (1993), «La phraséologie et son rôle dans l'enseignement/apprentissage d'une langue étrangère », Etudes de linguistique appliquée 92, pp. 82-113.

SCHAPIRA, C. (1999), Les stéréotypes en français : proverbes et autres formules, Paris, Ophrys.

SENELLART, J. (1998), «Reconnaissance automatique des entrées du lexique-grammaire des phrases figées ", in Lamiroy, B. (éd.) Le lexique-grammaire. Travaux de Linguistique, 37, pp. 109-127. 
